\title{
Temporal dynamics of the face familiarity effect: bootstrap analysis of single-subject event-related potential data
}

\author{
Esther Alonso-Prieto ${ }^{\mathrm{a}, \mathrm{b}, \mathrm{c} *}$, Raika Pancaroglu ${ }^{\mathrm{a}, \mathrm{c}}$, Kirsten A. Dalrymple ${ }^{\mathrm{a}, \mathrm{c}}$, Todd Handy ${ }^{\mathrm{d}}$, \\ Jason J. S. Barton ${ }^{\mathrm{a}, \mathrm{c}, \mathrm{d}}$ and Ipek Oruc ${ }^{\mathrm{a}, \mathrm{c}}$ \\ ${ }^{a}$ Department of Medicine (Neurology), University of British Columbia, Vancouver, BC, Canada; \\ ${ }^{b}$ VGH Eye Care Centre, Human Vision and Eye Movement Laboratory, third floor 2550 Willow Street, Vancouver, \\ BC, Canada V5Z 3N9; ${ }^{\circ}$ Department of Ophthalmology \& Visual Sciences, University of British Columbia, Vancouver, \\ BC, Canada; ${ }^{d}$ Department of Psychology, University of British Columbia, Vancouver, BC, Canada
}

(Manuscript received 23 July 2014; revised manuscript received 14 April 2015; revised manuscript accepted 17 May 2015)

Prior event-related potential studies using group statistics within a priori selected time windows have yielded conflicting results about familiarity effects in face processing. Our goal was to evaluate the temporal dynamics of the familiarity effect at all time points at the singlesubject level. Ten subjects were shown faces of anonymous people or celebrities. Individual results were analysed using a point-by-point bootstrap analysis. While familiarity effects were less consistent at later epochs, all subjects showed them between 130 and $195 \mathrm{~ms}$ in occipitotemporal electrodes. However, the relation between the time course of familiarity effects and the peak latency of the N170 was variable. We concluded that familiarity effects between 130 and $195 \mathrm{~ms}$ are robust and can be shown in single subjects. The variability of their relation to the timing of the N170 potential may lead to underestimation of familiarity effects in studies that use group-based statistics.

Keywords: face perception; familiarity; temporal dynamics; bootstrap; electroencephalography

Familiar faces can be recognized more accurately and faster than unfamiliar faces even under poor viewing conditions (see e.g., Bruce, Henderson, Newman, \& Burton, 2001; Burton, Wilson, Cowan, \& Bruce, 1999; Sinha, Balas, Ostrovsky, \& Russell, 2006; Tong \& Nakayama, 1999). The timing of the emergence of the neural processes that underlie this complex perceptual task is still not well understood. Previous studies have examined these mainly by using event-related potentials (ERPs), an electroencephalographic technique with high temporal resolution. The typical strategy has been to look for differences associated with viewing familiar versus unfamiliar faces in the amplitude of particular ERPs during specific time windows. However, these studies have not produced consistent results. Some reports found early face familiarity effects between 140 and $180 \mathrm{~ms}$ (during the N170 component) (Caharel, Courtay, Bernard, Lalonde, \& Rebai, 2005; Caharel, Fiori, Bernard, Lalonde, \& Rebai, 2006; Caharel et al., 2002; Harris \& Aguirre, 2008; Kloth et al., 2006; Marzi \& Viggiano, 2007; Todd, Lewis, Meusel, \& Zelazo, 2008; Wild-Wall, Dimigen, \& Sommer, 2008), but not others (Bentin \& Deouell, 2000; Eimer,

\footnotetext{
*Corresponding author. Email: e.alonso@eyecarecentre.org 
2000; Rossion et al., 1999), while yet others reported late face familiarity effects between 235 and $335 \mathrm{~ms}$ (N250 component; Tanaka, Curran, Porterfield, \& Collins, 2006).

Some of these discrepancies may be attributable to differences in experimental design, such as the type of familiarity of the faces used (e.g., personally familiar faces, in contrast to famous faces, may provide a richer face representation that encompasses not only visual but also strong emotional information). A less considered but equally important source of discrepant results may be the method of analysis. Two factors may be of particular concern. First, most previous reports contrasted peak or mean amplitude values over temporal windows that were selected a priori to include components such as the N170, which consistently show "face-sensitivity" (i.e., significant amplitude differences in response to faces compared to other objects). However, it is not logically necessary that the time periods when the neural signals generated by viewing faces and viewing objects differ the most will also be the periods when differences between familiar and unfamiliar faces will occur. Second, traditional ERP analysis relies on group statistics, by using as input the average data of individuals for each experimental condition and then comparing the effects of condition at a group level. These do not take into account individual differences in the timing of the emergence of familiarity effects and may potentially obscure interesting effects with dynamics that vary between single subjects constraining the range of conclusions possible.

To overcome such limitations, a major goal of this study was to implement an analytic method to study the electroencephalographic activity generated by familiar and unfamiliar faces time point by time point at the individual subject level. Such an analysis would be free of a priori assumptions about the particular time at which familiarity effects emerge and would still be sensitive to differences despite slight temporal variations in effects across subjects. The bootstrap technique is a statistical tool particularly suited for this kind of analysis. It creates a sampling distribution for the statistic of interest (e.g., mean) by resampling from the raw data (e.g., the amplitude values obtained at a particular time point in each trial) with replacement a large number of times (Efron \& Tibshirani, 1994; Wilcox, 2005; Wilcox \& Keselman, 2003). This sampling distribution can be used for hypothesis testing (Oruc et al., 2011). The bootstrap technique has been successfully used in ERP studies, for example, to establish the reliability of the locations of maxima in individual topographical maps (Fabiani, Gratton, Corballis, Cheng, \& Friedman, 1998), to determine the time at which the face sensitivity of particular components appears (Rousselet, Husk, Bennett, \& Sekuler, 2008), to permit statistical inferences about the presence or absence of face-sensitivity of the N170 in single patients (Dalrymple et al., 2011), and to generate ERP amplitude confidence intervals from two subgroups of subjects (Caryl, Golding, \& Hall, 1995). Therefore, in order to determine the presence of familiarity effects during the time window of the N170 component at the individual level, we implemented a point-by-point bootstrap technique, in which familiar and unfamiliar face viewing are compared at the individual level and at each selected electrode.

Although a valuable aspect of this method is that it is agnostic about when and where face familiarity effects will emerge, we considered an initial hypothesis. We hypothesized that the human face perception system can differentiate between familiar and unfamiliar faces as early as $130 \mathrm{~ms}$. This hypothesis is suggested by several previous findings. First, neurons in nonhuman primate inferotemporal cortex accumulate information about individual faces around $40-50 \mathrm{~ms}$ after information about the global category of faces have been coded (Matsumoto, Okada, Sugase-Miyamoto, Yamane, \& Kawano, 2005; Sugase, Yamane, Ueno, \& Kawano, 1999) and have an average onset latency of about $100 \mathrm{~ms}$ (Tovee \& Rolls, 1995). Second, adaptation studies in humans show that the amplitude of the face-sensitive N170 component (130$180 \mathrm{~ms}$ ) is reduced for identical faces compared to faces of different people (Jacques \& Rossion, 2006, 2007). Third, humans can discriminate faces behaviourally around $200 \mathrm{~ms}$ (Jacques \& Rossion, 2007). 


\section{Method}

\section{Subjects}

Ten healthy subjects (five males, mean age 28.1 \pm 3.57 years, all right-handed) participated in the study. All subjects had normal or corrected-tonormal visual acuity. The institutional review boards of Vancouver General Hospital and the University of British Columbia approved the protocol, and all subjects gave informed consent in accordance with the declaration of Helsinki.

\section{Stimuli}

We used images of faces slightly smiling or with neutral expression, without identifying accessories (e.g., glasses) and with only minimal hair visible (Figure 1). All images were sized to 400 pixels in width with a resolution of 300 pixels per inch. Four images were obtained for each of five different people in the two categories, familiar faces (pictures of celebrities) and unfamiliar faces (pictures of individuals unknown to the subjects). The small number of different identities in each category was used to avoid differences among the familiar faces in terms of degree of familiarity: The smaller the stimulus set, the higher the chances that all faces were equally familiar to all subjects. This was an important aspect to control for because even small differences in terms of degree of familiarity could have led to a decrease in the strength of the familiarity effect across trials pertaining to that condition. Variation in viewpoint from frontal to three-quarter view as well as slight differences in face sizes were used to minimize possible familiarity effects based on perceptual processing of visually identical retinal images (Figure 1).

Several measures were taken to minimize possible differences in low-level visual properties between the two picture sets (i.e., familiar and unfamiliar faces). First, the original pictures were converted to grey scale and were approximately equated for luminance and contrast using the SHINE toolbox (Willenbockel et al., 2010). We confirmed that there was no significant difference between the mean luminance of the familiar and unfamiliar face stimuli based on an independent-samples $t$-test (mean luminance $=8.74 \pm 1.94$ cd $\mathrm{m}^{-2}$ for familiar; $8.70 \pm 4.91 \mathrm{~cd} \mathrm{~m}^{-2}$ for unfamiliar), $t(38)=0.03, p=.98$. Similarly, there was no difference between average root mean square (RMS) contrast of the two stimulus sets (mean RMS contrast $=1.14 \pm 0.16$ for familiar and $1.28 \pm 0.32$ for unfamiliar), $t(38)=-1.81, p=.08$.

In addition, we performed a pilot study to determine whether we could detect effects due to lowlevel visual properties, by presenting the original pictures not equated for contrast and luminance to a different group of 11 subjects (mean age $28 \pm$ 10.12, three males, 9 right-handed). All aspects of the experimental procedure, electroencephalographic recording, and data analysis were similar to those of the actual experiment (described in detailed in the next sections) except that they were not warned in advance about whose faces they were going to see. On these data, we performed a point-by-point comparison using the bootstrap technique (see next section for further details on this technique) focusing on the P1 component. This component is a positive peak that reaches its highest amplitude between 80 and 120 ms at occipital and occipitotemporal electrodes. Previous face perception studies have shown that P1 amplitude modulations disappear (Rossion \& Caharel, 2011) or are weak in amplitude, scattered in time, and present in only a small minority of observers (Rousselet et al., 2008) once low-level properties are matched. Therefore, we reasoned that stimuli that differ in low-level properties should generate significant differences during the time window of this early P1 component, and that these P1 differences should be eliminated if the stimulus set is successfully equated for low-level properties. If the latter was achieved, then differences in later epochs would probably not be due to effects of low-level properties.

\section{Procedure}

A preexperimental questionnaire confirmed that all participants recognized the familiar faces and did not recognize the unfamiliar faces. This part of the protocol may also have enhanced our findings, as some authors have suggested that the N170 familiarity effect is more consistently observed if subjects know in advance the faces that are going to be presented (Caharel, Ramon, \& Rossion, 2014). 
Pilot Experiment
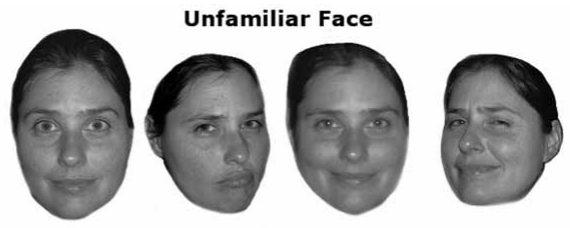

Familiar Face
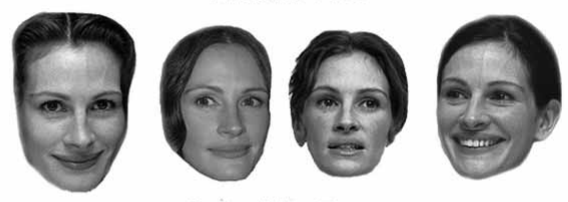

Final Experiment
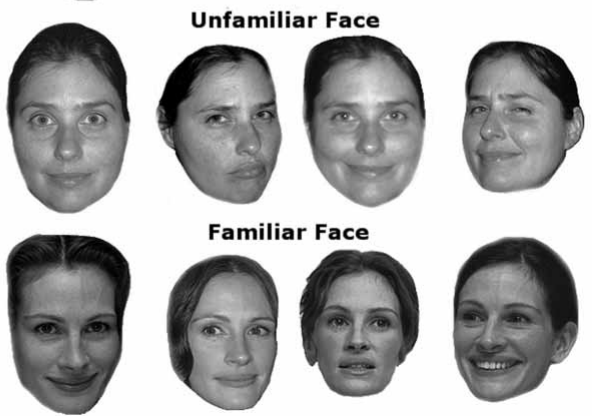

Figure 1. Examples of the faces used for both the pilot and the final experiment.

Subjects were seated in a sound-attenuated room, $100 \mathrm{~cm}$ from a $17^{\prime \prime}$ computer monitor. The experiment was programmed and carried out using MATLAB (Mathworks) and PsychToolbox (Brainard, 1997). Each trial began with a black fixation dot at the centre of the screen. Between 2700 and $2900 \mathrm{~ms}$ later a face, selected randomly from the familiar or unfamiliar categories, appeared for $100 \mathrm{~ms}$. It was followed by a 300 -ms visual mask. The next trial began with the reappearance of the fixation point. Subjects were instructed to indicate whether the images they saw were pleasant or unpleasant, by pressing a button on a video game controller as soon as possible. The response hand was counterbalanced across participants. This task was irrelevant to the purpose of the study and was implemented to ensure that subjects attended to the screen. There were 200 trials for each of the two categories, presented in a random order.

\section{Electrophysiological recording}

Brain electrical activity was recorded using a standard 64-electrode cap (Biosemi Active 2 system) and five additional electrodes (three eye movement channels plus the two mastoids). All recordings were performed relative to two scalp electrodes located over medial parietal cortex (CMS/DRL), amplified with a gain of 0.5 and digitized online at a sampling rate of 256 samples per second. Offsets at each active electrode, which are a running average of the voltage measured between CMS and each active electrode and reflect the half-cell potential of the electrode/gel/skin interface, were kept between $\pm 40 \mathrm{mV}$ at rest. Vertical eye movements were recorded using an electrode inferior to the right eye, while horizontal eye movements were recorded using electrodes on the right and left outer canthi.

\section{Analysis}

First, the data were visually inspected, and channels that were particularly noisy were identified, removed, and later interpolated using spherical splines as implemented in EEGLAB (Delorme \& Makeig, 2004). Each subject's signal was then algebraically transformed to average reference 
and low-pass filtered with a Butterworth filter (25.6 $\mathrm{Hz}$ half amplitude cut-off).

The bootstrap analysis of single-subject data was focused on a set of electrodes that, according to previous studies, exhibit the strongest and most consistent response to different types of visual stimuli, including faces. Those electrodes were: (a) P1/P2, P3/P4, P5/P6, P7/P8, P9/10, PO3/PO4, $\mathrm{PO} 7 / \mathrm{PO} 8, \mathrm{Pz}, \mathrm{POz}$, where the face-selective component N170 (see, for example, Jacques, d'Arripe, \& Rossion, 2007) as well as the N250 (see, for example, Tanaka et al., 2006) are most prominent; (b) $\mathrm{O} 1 / \mathrm{O} 2$ and $\mathrm{Oz}$, where early visual components (e.g., P1) usually reach their highest amplitude (see, for example, Di Russo, Martinez, Sereno, Pitzalis, \& Hillyard, 2002); (c) CPz, Cz, Fz, FCz, FT7/ FT8, FC5/FC6, FC3/FC4 and FC1/FC2, C1/C2, $\mathrm{CP} 1 / \mathrm{CP} 2$ where the positive counterpart of the N170, the VPP (Jeffreys, 1989, 1996; Joyce \& Rossion, 2005; Schendan, Ganis, \& Kutas, 1998), reaches its highest amplitude.

After each subject's data were preprocessed, trials were separated into the two categories of familiar and unfamiliar faces. Amplitude values were extracted at 4-ms intervals (corresponding to the sampling rate of the electroencephalography (EEG) equipment used) from 0 to $400 \mathrm{~ms}$ after stimulus onset (baseline correction was performed by subtracting the average amplitude over $100 \mathrm{~ms}$ of prestimulus activity from each time point). At each time point there was a data set of 200 amplitude values (one value for each of the 200 trials) for each of the two conditions for that subject. The bootstrap procedure resampled the data for a given time point with replacement 10,000 times. The average of each new resampled data set for familiar faces was subtracted from the mean of the new resampled data set for the unfamiliar faces. A histogram of these 10,000 differences between means was then created for each time point and each electrode separately. The lower and upper 2.5th percentile points of the histogram served as the critical values for two-tailed significance at the .05 level (see Oruc et al., 2011, for further details on the implementation of this method). A significant familiarity effect was one where the value 0 (i.e., no difference) lay outside these 2.5 th percentile points for the subtraction between familiar and unfamiliar conditions. For each subject we corrected for multiple comparisons using the Bonferroni correction adjusted for interitem correlations (Sankoh, Huque, \& Dubey, 1997). Note that this analysis is performed for each electrode individually, thus 100 bootstrap analyses per electrode, per subject were performed.

\section{Results}

\section{ERP components}

We first inspected the ERP response obtained from each individual subject to identify potentials denoted in earlier studies that have used a similar methodology (see, e.g., Rossion \& Jacques, 2011; Tanaka et al., 2006). We identified the P1 component as the most positive peak in the interval $80-120 \mathrm{~ms}$, the $\mathrm{N} 170$ as the most negative peak in the interval $130-190 \mathrm{~ms}$, the $\mathrm{P} 2$ as the most positive peak in the interval $190-275 \mathrm{~ms}$, and the N250 as the negative deflection in the interval 235-335 ms.

Components P1, N170, and P2 were observed in all 10 subjects. P1 reached its largest amplitude in occipital electrodes $\mathrm{O} 1$ and $\mathrm{O} 2$. N170 and P2 reached their largest amplitude in occipitotemporal electrodes (P7/P8, P9/P10, and PO7/PO8). Following component $\mathrm{P} 2$, a negative deflection, identified as the N250, was observed in six subjects. It reached its largest amplitude also at occipitotemporal electrodes (P7/P8, P9/P10, and PO7/PO8; Figure 2).

\section{The pilot experiment: Effects of matching luminance and contrast}

Using the pilot face stimuli that were not matched in luminance and contrast, we found that between 80 and $130 \mathrm{~ms}$, a period that coincided with the P1 component (Figure 3), differences between the familiar and unfamiliar sets appeared in three subjects in left occipitotemporal electrodes (P7, P9, and PO7), in eight subjects in occipital electrode O1. They also appeared in between one and eight subjects in right occipitotemporal electrodes (P8, $\mathrm{P} 10$, and PO8) and in six subjects in occipital electrode $\mathrm{O} 2$. In contrast, in the main experiment, in which luminance and contrast was matched across 


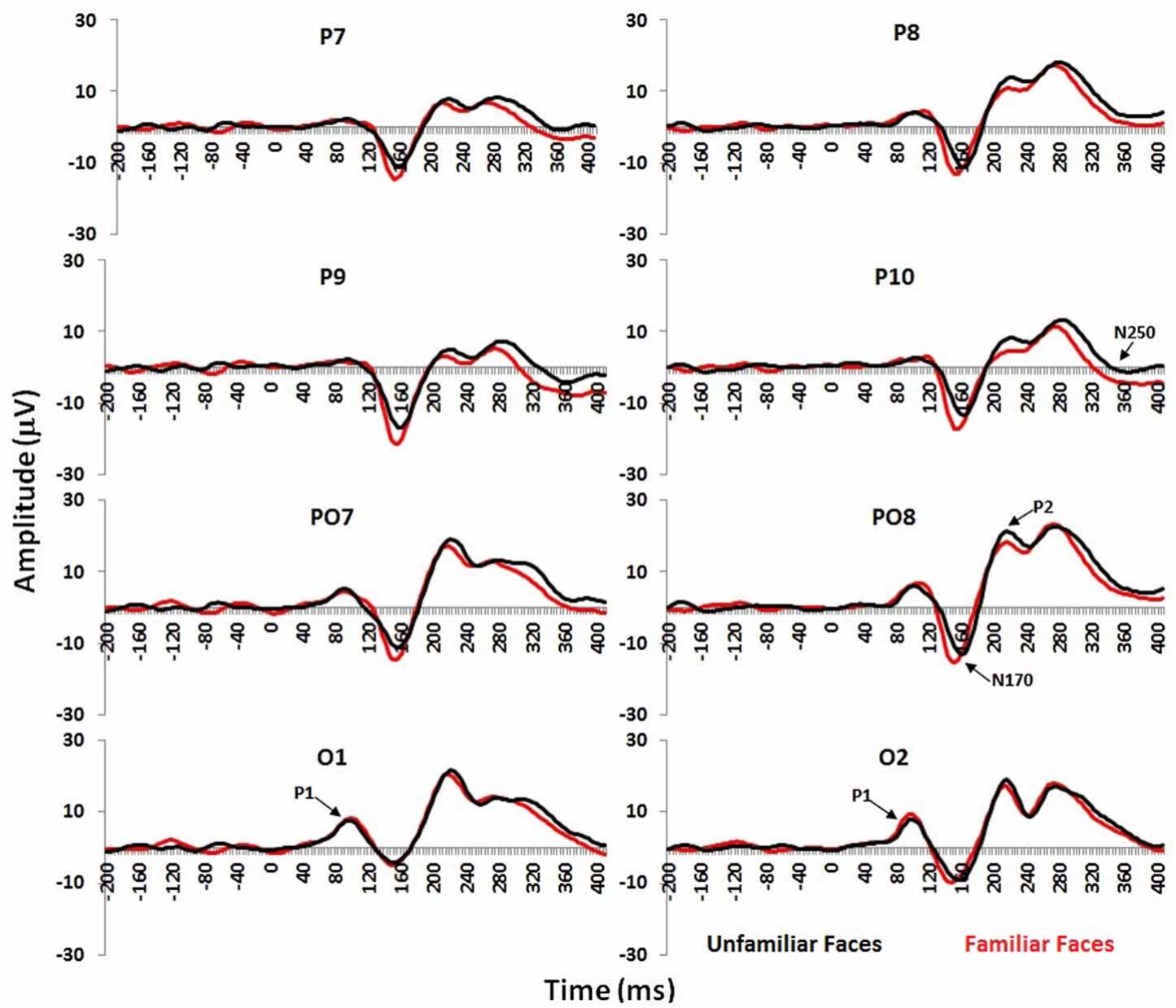

Figure 2. Averaged event-related potential (ERP) waveforms in response to familiar (red trace) and unfamiliar (black trace) faces in one representative participant. Components P1, N170, P2, and N250 components are indicated in the electrodes where they were most clearly observed. [To view this figure in colour, please see the online version of this Journal.]

the familiar and unfamiliar sets of faces (see Method), this early P1 effect was nearly eliminated, appearing only during scattered time points in three subjects: S01 showed P1 differences in electrodes PO7/PO8 and O1/O2, S05 only in PO8, and S10 only in P8 (Figure 3).

\section{Main experiment: Bootstrap results for familiarity effects}

We first determined the electrodes that exhibited the largest number of time points at which significant differences between familiar and unfamiliar conditions were detected across subjects. Considering that we had 100 time points (from 0 to $400 \mathrm{~ms}$ after stimulus onset with a 4-ms time resolution) and 10 subjects, the maximum number of time points for any given electrode was 1000 . The highest ranked electrodes were $\mathrm{P} 7 / \mathrm{P} 8, \mathrm{P} 9 / \mathrm{P} 10, \mathrm{PO} 7 / \mathrm{PO} 8$, and $\mathrm{Pz}$ (Figure 4), which, apart from $\mathrm{Pz}$, were also those with the largest N170, P2, and N250 components.

After selecting these as electrodes of interest, we determined when significant differences between conditions appeared for each subject. Significant 

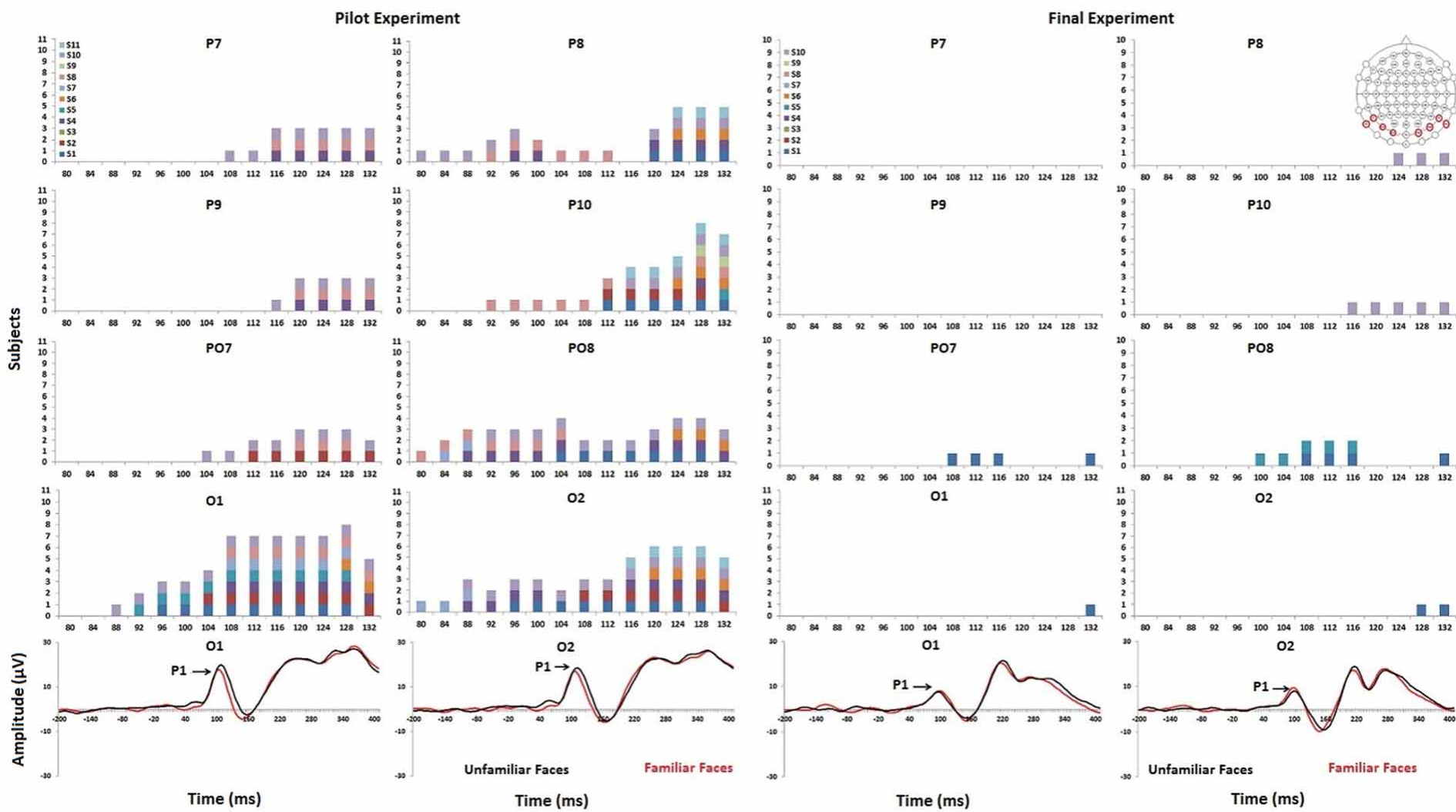

Figure 3. Differences between familiar and unfamiliar faces during the time window of the P1 component when the stimulus low-level properties were not equated (pilot experiment) and when they were equated (final experiment). For occipitotemporal and occipital electrodes, significant time points (80-130 ms) for familiarity effects are depicted as bars on a raster line. The bar colours indicate different subjects. Note that significant differences are more consistently observed when the stimulus low-level properties were not equated (pilot experiment). In the final experiment, for which such properties were equated across both stimulus sets, the differences between conditions are infrequent and scattered. Red circles indicate the scalp location of the electrodes of interest. [To view this figure in colour, please see the online version of this Journal.] 


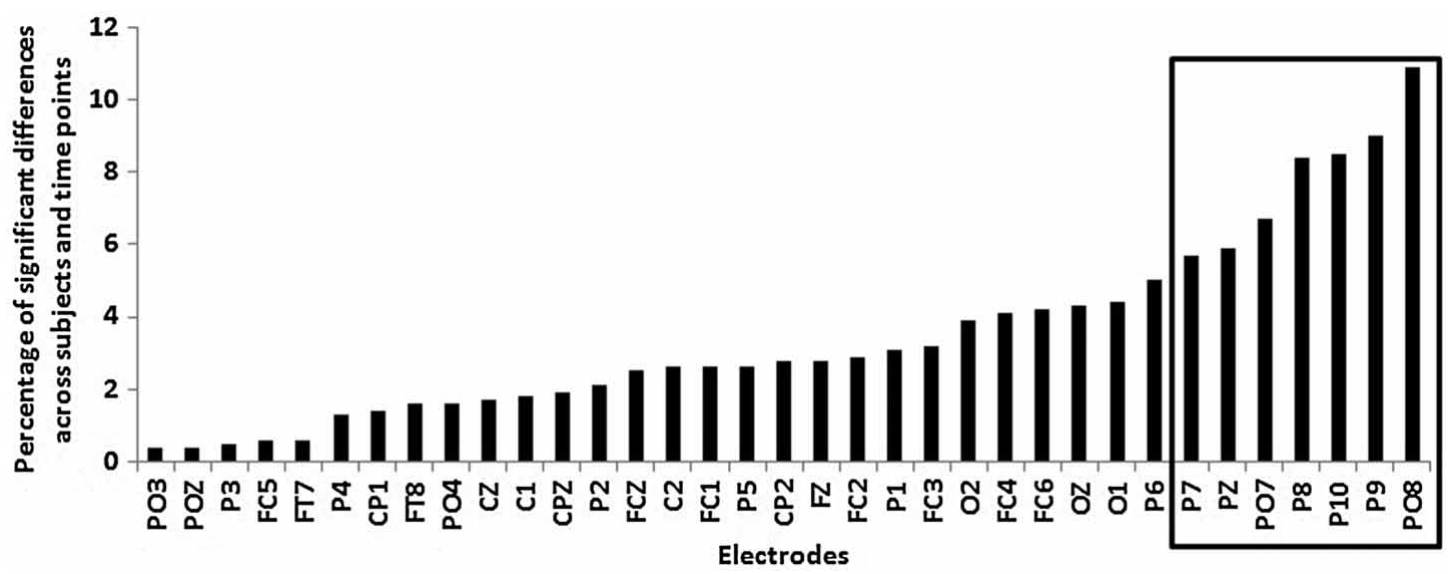

Figure 4. Percentage of significant differences found between familiar and unfamiliar face conditions across subjects and time points. Note that electrodes P7/P8, P9/P10, PO7/PO8, and Pz presented the highest amount of time points during which differences in the brain response to familiar and unfamiliar faces were found.

familiarity effects in electrode Pz were variable and only brief in six subjects: Its high ranking above was due to one anomalous subject ( $\mathrm{S} 02$ ) with prolonged late familiarity effects from about $260 \mathrm{~ms}$ onwards (Figure 5). Hence, to simplify our analysis, we limited further consideration of familiarity effects to $\mathrm{P} 7 / \mathrm{P} 8, \mathrm{P} 9 / \mathrm{P} 10$, and $\mathrm{PO} 7 / \mathrm{PO} 8$.

The most consistent familiarity effects were found between 130 and $195 \mathrm{~ms}$, coinciding with the N170 component. All 10 subjects showed familiarity effects in the right hemisphere and eight subjects in the left (Figure 5). Specifically, S01, 02, 04, 05, and 07 presented bilateral differences in all occipitotemporal electrodes of interest. S03, $06,08,09$, and 10 presented differences in at least one occipitotemporal electrode in the right hemisphere, while S06, 08, and 09 also presented differences in at least one occipitotemporal electrode in the left hemisphere. Differences in right occipital electrode $(\mathrm{O} 2)$ were observed in $\mathrm{S} 01,02,03,04$, 05,07 , and 08 . In the equivalent left-hemisphere electrode (O1) differences were observed in S01, 02, 05, 06, and 07 (Figure 5).

Later effects were more inconsistent. During the time window of the P2 (190-275 ms), three subjects showed familiarity effects in both hemispheres (S01, S02, S04), and one in the right hemisphere (S09; Figure 5). From 275 to $335 \mathrm{~ms}$, during the time window of the N250, three subjects (S04, $\mathrm{S} 07$, and S10) showed familiarity effects in both hemispheres, while one (S01) did in the right hemisphere only (Figure 5).

\section{Relation between familiarity effects and the N170 component}

The most consistent familiarity effects in our subjects thus appear to coincide with the time window of the N170 component. To better characterize the temporal relation between the N170 and familiarity effect in each subject, we noted the timing of the N170 peak amplitude and the beginning and end of the interval over which familiarity effects were found. The onset and offset of the familiarity effect in the 130-195-ms window in any of the P7/P8, $\mathrm{P} 9 / \mathrm{P} 10$, and PO7/PO8 electrodes was taken as the first appearance of a significant difference between familiar and unfamiliar conditions. The peak of the N170 in each subject was taken from a waveform averaged over the P7/P9/PO7 for the left and P8/ $\mathrm{P} 10 / \mathrm{PO} 8$ for the right hemisphere.

In four subjects (S04, S05, S07, and S08) the familiarity effect overlapped with the N170 peak (Figure 6). In the right hemisphere the familiarity effect began $11 \pm 8.25 \mathrm{~ms}$ before the N170 peak for familiar faces and ended $24 \pm 8.64 \mathrm{~ms}$ after, and began $23 \pm 13.22 \mathrm{~ms}$ before the N170 peak for unfamiliar faces and ended $12 \pm 11.78 \mathrm{~ms}$ after. In the left hemisphere, it began $10.00 \pm 4 \mathrm{~ms}$ before the N170 peak to familiar faces and ended 

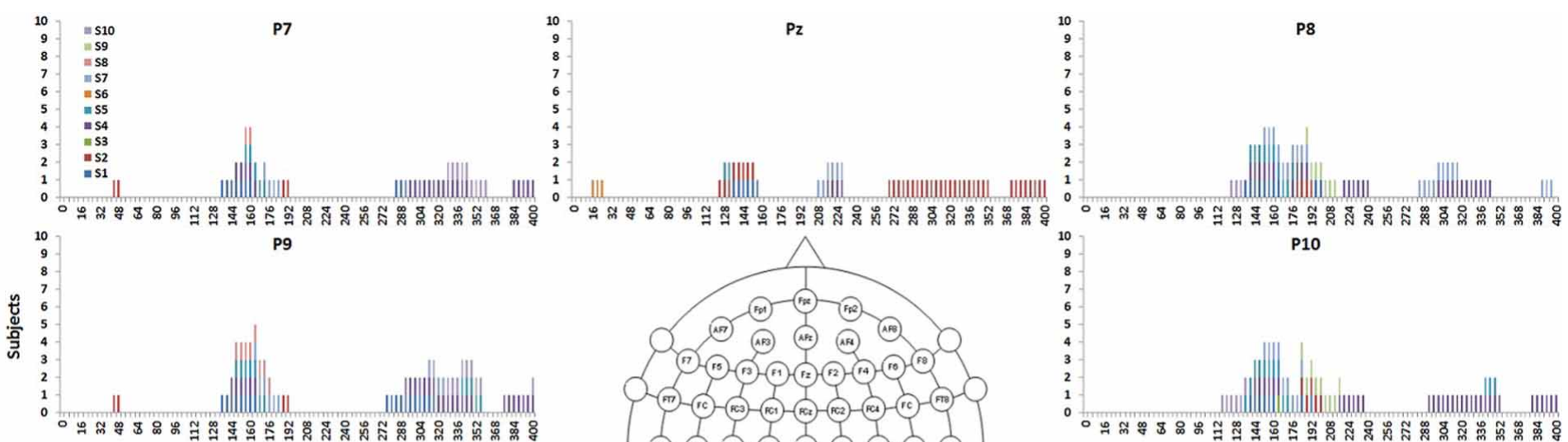
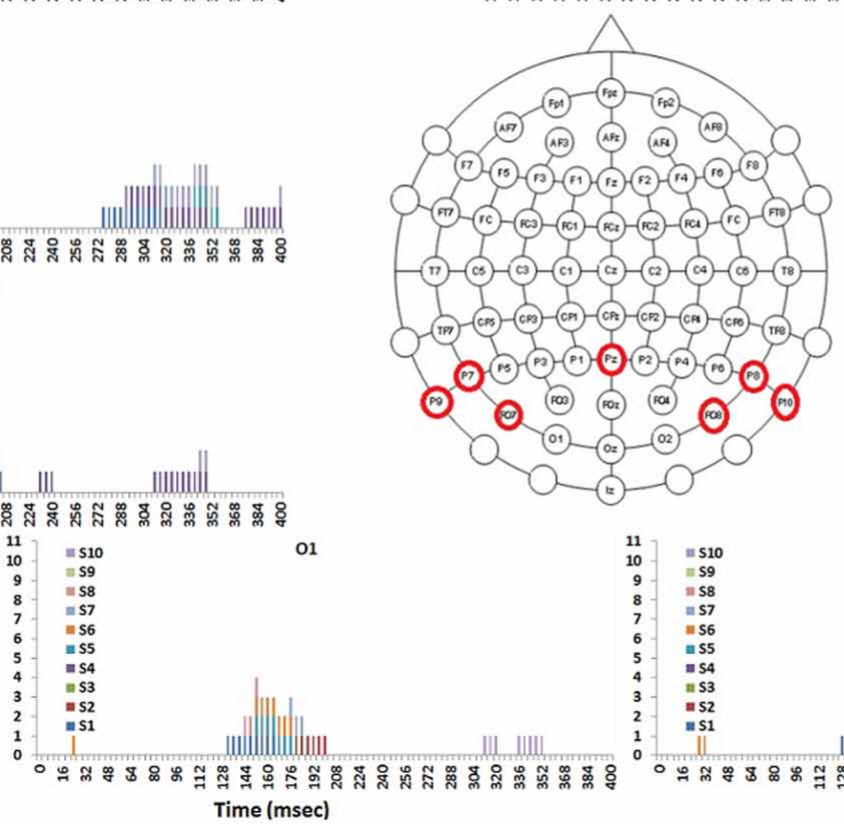

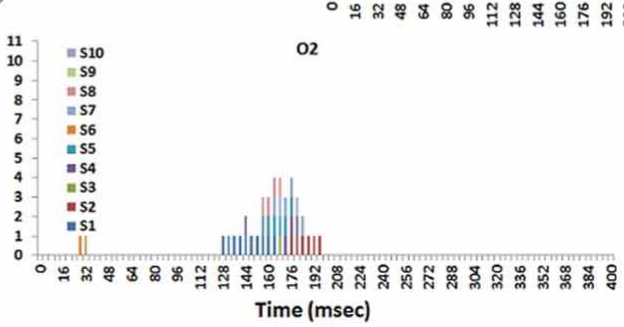

P10

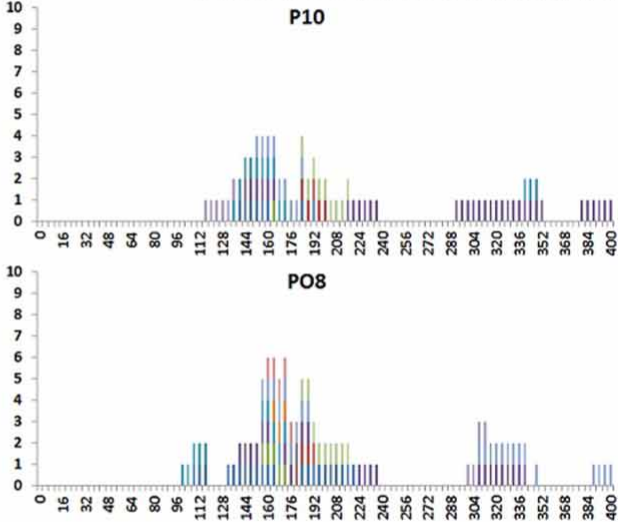

1
0
0
0
0
0
0
0
0
0
0
0
0
0
0

Figure 5. Individual bootstrap results: Each subject's significant time points for familiarity effects depicted as bars on a raster line. The bar colours indicated different subjects. Red circles indicate the scalp location of the electrodes of interest. Note that the differences were most consistently observed across subjects between 130 and $195 \mathrm{~ms}$. [To view this figure in colour, please see the online version of this Journal.] 


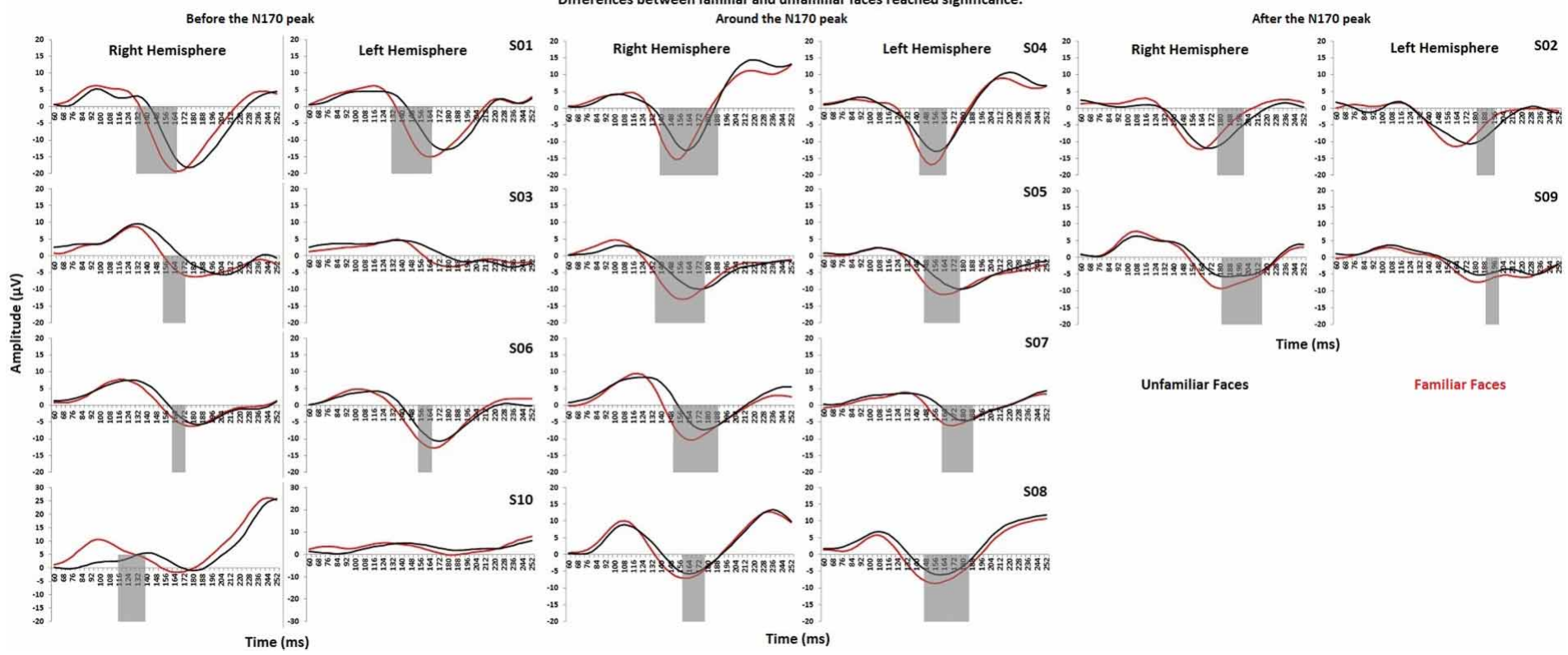

Figure 6. Temporal relation between the start and end of the familiarity effect and the N170 peak for each individual subject. For each participant, waveforms are presented from 60 to $250 \mathrm{~ms}$. Time intervals during which the difference between both waveforms reached statistical significance are indicated in grey. Subjects are grouped according to three patterns of temporal relation between the familiarity effect and the N170 peaks (see text for further details). [To view this figure in colour, please see the online version of this Journal.] 
$17 \pm 7.57 \mathrm{~ms}$ after, and began $18 \pm 9.52 \mathrm{~ms}$ before the N170 peak to unfamiliar faces and ended $9 \pm$ $11.49 \mathrm{~ms}$ after.

However, in four other subjects (S01, S03, S06, and S10) the familiarity effect ended before the N170 reached its peak. In the right hemisphere, it ended $13 \pm 12.81 \mathrm{~ms}$ before the N170 peak for familiar faces and $25 \pm 17.70 \mathrm{~ms}$ before the N170 peak for unfamiliar faces. In the left hemisphere, a familiarity effect was seen only in two of these four subjects, S01 and S06. In S01 it ended at the same time as the N170 peak to familiar faces and $12 \mathrm{~ms}$ before the peak to unfamiliar faces, while in S06 it ended $8 \mathrm{~ms}$ before the N170 peak to familiar faces and $12 \mathrm{~ms}$ before the N170 peak to unfamiliar faces.

Finally, in two subjects (S02 and S09) the effect began after the N170 peak. In the right hemisphere, S02 had a familiarity effect that began $16 \mathrm{~ms}$ after the N170 peak to familiar faces and $8 \mathrm{~ms}$ after the N170 peak to unfamiliar faces, while in S09 it began $4 \mathrm{~ms}$ after the N170 peak to familiar faces and at the same time as the N170 peak to unfamiliar faces. In the left hemisphere, in the familiarity effect began $20 \mathrm{~ms}$ after the N170 peak to familiar faces and $8 \mathrm{~ms}$ after the N170 peak to unfamiliar faces, while in S09 it began $12 \mathrm{~ms}$ after the N170 peak to familiar faces and $8 \mathrm{~ms}$ after the N170 peak to unfamiliar faces.

Inspection of the individual traces revealed that the most consistent effect of familiarity is a shift of part or all of the N170 waveform towards earlier time points (Figure 6). Whether such a shift translates to a difference between the two curves at a particular time of the N170 potential depends on a number of factors, including the size of the shift, whether the shift affects both the descending and ascending arms of the waveform similarly or if there is a change in the width of the potential, and whether there is also a change in the amplitude of the peak, as appears to be the case in only some subjects (e.g., S04, S05, and S07).

\section{Discussion}

We used a point-by-point bootstrap analysis to study the temporal dynamics of the face familiarity effects at the single-subject level. This method avoids a priori assumptions about the temporal window of the effect and accounts for interindividual differences. Most consistently, we found familiarity effects in all participants in occipitotemporal electrodes of the right hemisphere and in eight participants in the left hemisphere between 130 and 195 ms, coinciding with the N170 component. However, we also found that the temporal relation between familiarity effects and the peak latency of the N170 component differs between subjects, sometimes before, after, or coinciding with the peak.

Before we discuss our results, a number of technical factors deserve mention. First, we used a small number of different identities (five identities, four versions of each one). This could have two consequences: Subjects might become familiar with the unfamiliar faces over the course of the experiment, or our familiarity effects could be confounded by repetition effects. The first of these, increasing familiarity with the unfamiliar set, might have reduced the difference in familiarity effects between the familiar and unfamiliar set: If anything this would bias against the positive results we obtained. Regarding the repetition effect, previous ERP studies using a wide variety of stimuli have shown that repeated items generate more positive waveforms, but that these occur more consistently between 250 and 500 ms (Doyle \& Rugg, 1998; Friedman, 1990; Mecklinger \& Meinshausen, 1998; Munte et al., 1997; Nessler, Mecklinger, \& Penney, 2005; Penney, Mecklinger, \& Nessler, 2001; Petten, Kutas, Kluender, Mitchiner, \& McIsaac, 1991; Rugg \& Doyle, 1992; Rugg \& Nagy, 1989), a latency value that has been replicated using faces (Jemel, Calabria, Delvenne, Crommelinck, \& Bruyer, 2003; Munte et al., 1997; Nessler et al., 2005) and is later than the key familiarity effects we describe. Furthermore, the number of repetitions of each image is the same for both our unfamiliar and familiar set: Hence any repetition effect based upon image properties should be the same for both conditions. A second issue that can confound familiarity effects is the new/old or novelty effect, differences generated by the appearance of a stimulus not seen before. To guard against this, we had subjects see in advance all the faces that were going to be presented. Also, novelty effects in ERP appear about 300 to $800 \mathrm{~ms}$ after stimulus onset (see Friedman \& Johnson, 2000; Mecklinger, 2000, for a 
review; Rugg, 1995), with an early midfrontal negative ERP effect between 300 and $500 \mathrm{~ms}$ and a later parietal positive ERP between 400 and $800 \mathrm{~ms}$ (Curran, 2000; Friedman \& Johnson, 2000; Mecklinger, 2000; Rugg, 1995). Thus, these novelty effects have a different topography and a longer latency later than the familiarity effects we found.

A third technical issue is whether other differences between the two stimulus sets besides familiarity could confound the results, in particular differences in low-level visual properties such as luminance and contrast. We controlled for this physically by equating both sets of face stimuli for luminance and contrast and statistically verifying that the match was successful. We then performed a pilot experiment to show that this process nearly eliminated any difference in the evoked response to the two stimulus sets in the early P1 component, which covers a period of activity highly sensitive to changes in low-level visual factors (Anllo-Vento \& Hillyard, 1996; MacKay \& Jeffreys, 1973; Shaw \& Cant, 1980; Yiannikas \& Walsh, 1983). As a consequence, we reduced the likelihood that any difference in later potentials in our main experiment with matched face stimuli were due to low-level image properties.

\section{Face familiarity effects during the time window of the N170 component}

Previous studies of face familiarity effects in the N170 have yielded conflicting results. Familiarity modulations of this component have been found with personally familiar faces, such as those of a subject's mother, their friends, or their own self (Caharel et al., 2006; Caharel et al., 2002; Kloth et al., 2006), famous faces (Caharel et al., 2006; Caharel et al., 2002) or recently learned faces associated with semantic information (Heisz \& Shedden, 2009). However, this has not been replicated in other studies that have also used personally familiar or famous faces (Bentin \& Deouell, 2000; Eimer, 2000; Henson et al., 2003; Jemel, Calabria, et al., 2003; Jemel, Pisani, Calabria, Crommelinck, \& Bruyer, 2003; Jemel, Pisani, Rousselle, Crommelinck, \& Bruyer, 2005) or recently learned faces (Rossion et al., 1999). A number of factors have been proposed to explain these discrepancies (e.g., type of face familiarity studied, number of times an unfamiliar face is presented throughout the experiment, type of task, etc.).

Our study revealed that an additional factor to consider is the intersubject variability regarding the temporal relationship between the emergence of the familiarity effect and the peak latency of the N170 component. In less than half of our subjects did the period with statistically significant differences between familiar and unfamiliar faces overlap with the N170 peak: In the rest it either preceded or followed the peak. The chief effect of familiarity appeared to be a shift of the N170 waveform to an earlier occurrence. How much of a shift occurs and whether it is accompanied by changes to the shape of the N170 potential determines when statistically significant familiarity effects will be found. These individual differences may arise from dissimilarities across subjects in nonfunctional factors such as skull thickness and cortical folding patterns, which influence the morphology of the waveforms. They may also arise from cognitive factors such as the speed at which evidence accumulates for a decision about familiarity. Regardless, this variability has methodological implications for group analyses. If the window of analysis is too narrowly centred around the N170 peak, a substantial number of subjects may not show familiarity effects in that window, reducing the sensitivity of a group-based analysis. Furthermore, our results indicate that a window focused on the ascending limb of the N170 potential has the greatest sensitivity to familiarity effects.

Familiarity effects beyond $190 \mathrm{~ms}$ were observed in only a minority of subjects using the bootstrap technique. This result does not exclude the possibility that familiarity effect may emerge beyond $190 \mathrm{~ms}$ - for example, during the N250 component-since several reasons may explain why we did not find such effects here. First, the bootstrap method is less sensitive when the component is not well differentiated (see, for example, S10 in Figure 6) or encompasses a broad waveform, which is characteristic of late cognitive potentials (Oruc et al., 2011). Second, our protocol had our subjects produce a motor response, which could have obscured the N250 and P3 components. Previous studies have shown that the P3 and N2 (the 
$\mathrm{N} 250$ is considered part of the N2 family of negative deflections at 200-300 ms after stimulus onset, see, e.g., Warren, Tanaka, \& Holroyd, 2011) are sensitive to response production. The P3 increases at parietal and decreases at the vertex and frontal electrodes when a stimuli demands a response, a "go-stimulus". Likewise, a "go-stimulus" decreases the N2 amplitude independent of the type of response (Pfefferbaum, Ford, Weller, \& Kopell, 1985). The net effect of having a motor response would then be a decreased signal-to-noise ratio in these temporal windows, so that familiarity modulations are not strong enough to be detected. Indeed, problems with signal-to-noise ratio in general for these late potentials may underlie the fact that some previous studies only found face familiarity effects in the $\mathrm{N} 250$ potential after averaging together several channels to increase the signal-tonoise ratio (see e.g., Tanaka et al., 2006).

\section{Conclusions}

In summary, our study shows that consistent differences between familiar and unfamiliar faces can be found at the single-subject level between 130 and $195 \mathrm{~ms}$. This has two implications. First, there is sufficient perceptual encoding by this time period to differentiate facial identity. Second, there is already modulation of such processing by stored information of previously seen faces at this early stage. However, the variable relationship between the N170 component and the timing of familiarity effects between subjects suggests that perceptual encoding and familiarity modulation for faces may be distinct. At a practical level, this variability highlights the value of the bootstrap technique for single-subject analyses that eliminate the need for and potential bias of a priori selection of temporal windows of analysis and has important implications for group-based analysis. First, it may provide an explanation that reconciles the mixed results of prior group analyses: Whether a significant familiarity effect is found in the N170 potential in any given study may depend upon the mix of individuals that comprise the group and the degree of homogeneity of their individual familiarity effects. Also following from this would be an important cautionary note: Heterogeneity could result in a null effect at a group level, even when familiarity effects are significant at the individual subject level.

\section{Disclosure statement}

No potential conflict of interest was reported by the authors.

\section{Funding}

This work was supported by the Canadian Institutes of Health Research (CIHR) [grant number MOP-106511]. Jason J. Barton was supported by a Canada Research Chair. Ipek Oruc was supported by a Natural Sciences and Engineering Research Council of Canada (NSERC) Discovery Grant [grant number RGPIN 402654-11].

\section{References}

Anllo-Vento, L., \& Hillyard, S. A. (1996). Selective attention to the color and direction of moving stimuli: electrophysiological correlates of hierarchical feature selection. Perception \& Psychophysics, 58, 191-206.

Bentin, S., \& Deouell, L. Y. (2000). Structural encoding and identification in face processing: ERP evidence for separate mechanisms. Cognitive Neuropsychology, 17, 35-55. doi: 10.1080/026432900380472

Brainard, D. H. (1997). The psychophysics toolbox. Spatial Vision, 10, 433-436.

Bruce, V., Henderson, Z., Newman, C., \& Burton, A. M. (2001). Matching identities of familiar and unfamiliar faces caught on CCTV images. Journal of Experimental Psychology: Applied, 7, 207-218. doi: 10.1037/1076-898x.7.3.207

Burton, A. M., Wilson, S., Cowan, M., \& Bruce, V. (1999). Face recognition in poor-quality video: Evidence from security surveillance. Psychological Science, 10, 243-248. doi: 10. 1111/1467-9280.00144

Caharel, S., Courtay, N., Bernard, C., Lalonde, R., $\&$ Rebai, M. (2005). Familiarity and emotional expression influence an early stage of face processing: An electrophysiological study. Brain and Cognition, 59, 96-100. doi: 10.1016/j. bandc.2005.05.005

Caharel, S., Fiori, N., Bernard, C., Lalonde, R., \& Rebai, M. (2006). The effects of inversion and 
eye displacements of familiar and unknown faces on early and late-stage ERPs. International Journal of Psychophysiology, 62, 141-151. doi: S0167-8760(06)00072-9 [pii] 10.1016/j.ijpsycho.2006.03.002

Caharel, S., Poiroux, S., Bernard, C., Thibaut, F., Lalonde, R., \& Rebai, M. (2002). ERPs associated with familiarity and degree of familiarity during face recognition. International Journal of Neuroscience, 112, 1499-1512. doi: 10. 1080/00207450290158368

Caharel, S., Ramon, M., \& Rossion, B. (2014). Face familiarity decisions take $200 \mathrm{msec}$ in the human brain: Electrophysiological evidence from a go/no-go speeded task. Journal of Cognitive Neuroscience, 26, 81-95. doi: 10. 1162/Jocn_a_00451

Caryl, P. G., Golding, S. J. J., \& Hall, B. J. D. (1995). Interrelationships among auditory and visual cognitive tasks: An event-related potential (ERP) study. Intelligence, 21, 297-326. doi: 10.1016/0160-2896(95)90019-5

Curran, T. (2000). Brain potentials of recollection and familiarity. Memory and Cognition, 28, 923-938.

Dalrymple, K. A., Oruc, I., Duchaine, B., Pancaroglu, R., Fox, C. J., Iaria, G., ... Barton, J. J. (2011). The anatomic basis of the right face-selective N170 IN acquired prosopagnosia: a combined ERP/fMRI study. Neuropsychologia, 49, 2553-2563. doi: 10. 1016/j.neuropsychologia.2011.05.003

Delorme, A., \& Makeig, S. (2004). EEGLAB: An open source toolbox for analysis of single-trial EEG dynamics including independent component analysis. Journal of Neuroscience Methods, 134, 9-21. doi: 10.1016/j.jneumeth. 2003.10.009

Di Russo, F., Martinez, A., Sereno, M. I., Pitzalis, S., \& Hillyard, S. A. (2002). Cortical sources of the early components of the visual evoked potential. Human Brain Mapping, 15, 95-111.

Doyle, M. C., \& Rugg, M. D. (1998). Word repetition within- and across-visual fields: An event-related potential study. Neuropsychologia, 36, 14031415.

Efron, B., \& Tibshirani, R. J. (1993). An introduction to the bootstrap. New York: Chapman and Hall.
Eimer, M. (2000). Event-related brain potentials distinguish processing stages involved in face perception and recognition. Clinical Neurophysiology, 111, 694-705. doi: 10.1016/ S1388-2457(99)00285-0

Fabiani, M., Gratton, G., Corballis, P., Cheng, J., \& Friedman, D. (1998). Bootstrap assessment of the reliability of maxima in surface maps of brain activity of individual subjects derived with electrophysiological and optical methods. Behavior Research Methods, Instruments, \& Computers, 30, 78-86. doi: 10.3758/bf03209418

Friedman, D. (1990). ERPs during continuous recognition memory for words. Biological Psychology, 30, 61-87.

Friedman, D., \& Johnson, R. Jr. (2000). Eventrelated potential (ERP) studies of memory encoding and retrieval: A selective review. Microscopy Research and Technique, 51, 6-28. doi: 10.1002/1097-0029(20001001)51: $1<6::$ AID-JEMT2>3.0.CO;2-R

Harris, A. M., \& Aguirre, G. K. (2008). The effects of parts, wholes, and familiarity on face-selective responses in MEG. Journal of Vision, 8. doi: Artn4Doi10.1167/8.10.4

Heisz, J. J., \& Shedden, J. M. (2009). Semantic learning modifies perceptual face processing. Journal of Cognitive Neuroscience, 21, 11271134. doi: 10.1162/jocn.2009.21104

Henson, R. N., Goshen-Gottstein, Y., Ganel, T., Otten, L. J., Quayle, A., \& Rugg, M. D. (2003). Electrophysiological and haemodynamic correlates of face perception, recognition and priming. Cerebral Cortex, 13, 793-805. doi: 10. 1093/cercor/13.7.793

Jacques, C., d'Arripe, O., \& Rossion, B. (2007). The time course of the inversion effect during individual face discrimination. Journal of Vision, 7, 3. doi: 10.1167/7.8.3

Jacques, C., \& Rossion, B. (2006). The speed of individual face categorization. Psychological Science, 17, 485-492. doi: 10.1111/j.14679280.2006.01733.x

Jacques, C., \& Rossion, B. (2007). Early electrophysiological responses to multiple face orientations correlate with individual discrimination performance in humans. Neuroimage, 36, 863876. doi: 10.1016/j.neuroimage.2007.04.016 
Jeffreys, D. A. (1989). A face-responsive potential recorded from the human scalp. Experimental Brain Research, 78, 193-202.

Jeffreys, D. A. (1996). Evoked potential studies of face and object processing. Visual Cognition, 3, 1-38. doi: 10.1080/713756729

Jemel, B., Calabria, M., Delvenne, J. F., Crommelinck, M., \& Bruyer, R. (2003). Differential involvement of episodic and face representations in ERP repetition effects. Neuroreport, 14, 525-530. doi: 10.1097/01. wnr.0000057864.05120.ba

Jemel, B., Pisani, M., Calabria, M., Crommelinck, M., \& Bruyer, R. (2003). Is the N170 for faces cognitively penetrable? Evidence from repetition priming of Mooney faces of familiar and unfamiliar persons. Cognitive Brain Research, 17, 431-446. doi: 10.1016/S09266410(03)00145-9

Jemel, B., Pisani, M., Rousselle, L., Crommelinck, M., \& Bruyer, R. (2005). Exploring the functional architecture of person recognition system with event-related potentials in a within- and cross-domain self-priming of faces. Neuropsychologia, 43, 2024-2040. doi: 10.1016/j.neuropsychologia.2005.03.016

Joyce, C., \& Rossion, B. (2005). The face-sensitive N170 and VPP components manifest the same brain processes: The effect of reference electrode site. Clinical Neurophysiology, 116, 2613-2631. doi: 10.1016/j.clinph.2005.07.005

Kloth, N., Dobel, C., Schweinberger, S. R., Zwitserlood, P., Bolte, J., \& Junghofer, M. (2006). Effects of personal familiarity on early neuromagnetic correlates of face perception. European Journal of Neuroscience, 24, 33173321. doi: 10.1111/j.1460-9568.2006.05211.x

MacKay, D. M., \& Jeffreys, D. A. (1973). Visually evoked potentials and visual perception in man. In R. Jung (Ed.), Handbook of sensory physiology (pp. 674-678). Berlin: Springer-Verlag.

Marzi, T., \& Viggiano, M. P. (2007). Interplay between familiarity and orientation in face processing: An ERP study. International Journal of Psychophysiology, 65, 182-192. doi: 10. 1016/j.ijpsycho.2007.04.003
Matsumoto, N., Okada, M., Sugase-Miyamoto, Y., Yamane, S., \& Kawano, K. (2005). Population dynamics of face-responsive neurons in the inferior temporal cortex. Cerebral Cortex, 15, 1103-1112. doi: 10.1093/cercor/bhh209

Mecklinger, A. (2000). Interfacing mind and brain: A neurocognitive model of recognition memory. Psychophysiology, 37, 565-582.

Mecklinger, A., \& Meinshausen, R. M. (1998). Recognition memory for object form and object location: An event-related potential study. Memory and Cognition, 26, 1068-1088.

Munte, T. F., Brack, M., Grootheer, O., Wieringa, B. M., Matzke, M., \& Johannes, S. (1997). Event-related brain potentials to unfamiliar faces in explicit and implicit memory tasks. Neuroscience Research, 28, 223-233.

Nessler, D., Mecklinger, A., \& Penney, T. B. (2005). Perceptual fluency, semantic familiarity and recognition-related familiarity: An electrophysiological exploration. Cognitive Brain Research, 22, 265-288. doi: 10.1016/j.cogbrainres.2004. 03.023

Oruc, I., Krigolson, O., Dalrymple, K., Nagamatsu, L. S., Handy, T. C., \& Barton, J. J. S. (2011). Bootstrap analysis of the single subject with event related potentials. Cognitive Neuropsychology, 28, 322-337. doi: 10.1080/ 02643294.2011 .648176

Penney, T. B., Mecklinger, A., \& Nessler, D. (2001). Repetition related ERP effects in a visual object target detection task. Cognitive Brain Research, 10, 239-250.

Petten, C. V., Kutas, M., Kluender, R., Mitchiner, M., \& McIsaac, H. (1991). Fractionating the word repetition effect with event-related potentials. Journal of Cognitive Neuroscience, 3, 131-150. doi: 10.1162/jocn.1991.3.2.131

Pfefferbaum, A., Ford, J. M., Weller, B. J., \& Kopell, B. S. (1985). Erps to response production and inhibition. Electroencephalography and Clinical Neurophysiology, 60, 423-434. doi: 10.1016/0013-4694(85)91017-X

Rossion, B., \& Caharel, S. (2011). ERP evidence for the speed of face categorization in the human brain: Disentangling the contribution of low-level visual cues from face perception. 
Vision Research, 51, 1297-1311. doi: 10.1016/ j.visres.2011.04.003

Rossion, B., Campanella, S., Gomez, C. M., Delinte, A., Debatisse, D., Liard, L., ... Guerit, J-M. (1999). Task modulation of brain activity related to familiar and unfamiliar face processing: An ERP study. Clinical Neurophysiology, 110, 449-462. doi: 10.1016/ S1388-2457(98)00037-6

Rossion, B., \& Jacques, C. (2011). The N170: understanding the time course of face perception in the human brain. In S. Luck \& E. Kappenman (Eds.), The Oxford handbook of ERP components (pp. 115-142). Oxford, UK: Oxford University Press.

Rousselet, G. A., Husk, J. S., Bennett, P. J., \& Sekuler, A. B. (2008). Time course and robustness of ERP object and face differences. Journal of Vision, 8, 3. 1-18. doi: 10.1167/8. 12.3

Rugg, M. D. (1995). ERP studies of memory. In M. D. Rugg \& M. G. H. Coles (Eds.), Electrophysiology of mind (pp. 132-170). New York: Oxford Univ Press.

Rugg, M. D., \& Doyle, M. C. (1992). Event-related potentials and recognition memory for low- and high-frequency words. Journal of Cognitive Neuroscience, 4, 69-79. doi: 10.1162/jocn. 1992.4.1.69

Rugg, M. D., \& Nagy, M. E. (1989). Event-related potentials and recognition memory for words. Electroencephalography and Clinical Neurophysiology, 72, 395-406.

Sankoh, A. J., Huque, M. F., \& Dubey, S. D. (1997). Some comments on frequently used multiple endpoint adjustments methods in clinical trials. Statistics in Medicine, 16, 2529-2542.

Schendan, H. E., Ganis, G., \& Kutas, M. (1998). Neurophysiological evidence for visual perceptual categorization of words and faces within 150 ms. Psychophysiology, 35, 240-251. doi: 10.1017/S004857729897010x

Shaw, N. A., \& Cant, B. R. (1980). Age-dependent changes in the latency of the pattern visual evoked potential. Electroencephalography and Clinical Neurophysiology, 48, 237-241.

Sinha, P., Balas, B., Ostrovsky, Y., \& Russell, R. (2006). Face recognition by humans:
Nineteen results all computer vision researchers should know about. Proceedings of the IEEE, 94, 1948-1962. doi: 10.1109/Jproc. 2006.884093

Sugase, Y., Yamane, S., Ueno, S., \& Kawano, K. (1999). Global and fine information coded by single neurons in the temporal visual cortex. Nature, 400, 869-873. doi: 10.1038/23703

Tanaka, J. W., Curran, T., Porterfield, A. L., \& Collins, D. (2006). Activation of preexisting and acquired face representations: The N250 event-related potential as an index of face familiarity. Journal of Cognitive Neuroscience, 18 , 1488-1497. doi: 10.1162/jocn.2006.18.9.1488

Todd, R. M., Lewis, M. D., Meusel, L. A., \& Zelazo, P. D. (2008). The time course of social-emotional processing in early childhood: ERP responses to facial affect and personal familiarity in a Go-Nogo task. Neuropsychologia, 46, 595613. doi: 10.1016/j.neuropsychologia.2007. 10.011

Tong, F., \& Nakayama, K. (1999). Robust representations for faces: Evidence from visual search. Journal of Experimental Psychology: Human Perception and Performance, 25, 1016-1035. doi: 10.1037//0096-1523.25.4.1016

Tovee, M., \& Rolls, E. T. (1995). Information encoding in short firing rate epochs by single neurons in the primate temporal visual cortex. Visual Cognition, 2, 35-58.

Warren, C. M., Tanaka, J. W., \& Holroyd, C. B. (2011). What can topology changes in the oddball N2 reveal about underlying processes? Neuroreport, 22, 870-874. doi: 10.1097/Wnr. 0b013e32834bbe1f

Wilcox, R. R. (2005). An affine invariant rankbased method for comparing dependent groups. The British Journal Of Mathematical and Statistical Psychology, 58, 33-42. doi: 10. 1348/000711005X47177

Wilcox, R. R., \& Keselman, H. J. (2003). Modern robust data analysis methods: Measures of central tendency. Psychological Methods, 8, 254-274. doi: 10.1037/1082-989X.8.3.254

Wild-Wall, N., Dimigen, O., \& Sommer, W. (2008). Interaction of facial expressions and familiarity: ERP evidence. Biological Psychology, 77, 138149. doi: 10.1016/j.biopsycho.2007.10.001 
Willenbockel, V., Sadr, J., Fiset, D., Horne, G. O., Gosselin, F., \& Tanaka, J. W. (2010). Controlling low-level image properties: The SHINE toolbox. Behavior Research Methods, 42, 671-684. doi: 10.3758/BRM.42.3.671
Yiannikas, C., \& Walsh, J. C. (1983). The variation of the pattern shift visual evoked response with the size of the stimulus field. Electroencephalography and Clinical Neurophysiology, 55, 427-435. 